\title{
On the structure of self-gravitating molecular clouds
}

\author{
V. Ossenkopf ${ }^{1}$, R. S. Klessen ${ }^{2,3}$, and F. Heitsch ${ }^{3}$ \\ 1 1. Physikalisches Institut, Universität zu Köln, Zülpicher Straße 77, 50937 Köln, Germany \\ e-mail: ossk@ph1.uni-koeln.de \\ 2 UCO/Lick Observatory, University of California, Santa Cruz, CA 95064, USA \\ e-mail: ralf@ucolick.org \\ 3 Max-Planck-Institut für Astronomie, Königstuhl 17, 69117 Heidelberg, Germany \\ e-mail: heitsch@mpia-hd.mpg.de
}

Received 16 July 2001 / Accepted 17 September 2001

\begin{abstract}
To study the interaction of star-formation and turbulent molecular cloud structuring, we analyse numerical models and observations of self-gravitating clouds using the $\Delta$-variance as statistical measure for structural characteristics. In the models we resolve the transition from purely hydrodynamic turbulence to gravitational collapse associated with the formation and mass growth of protostellar cores. We compare models of driven and freely decaying turbulence with and without magnetic fields. Self-gravitating supersonic turbulence always produces a density structure that contains most power on the smallest scales provided by collapsed cores as soon as local collapse sets in. This is in contrast to non-self-gravitating hydrodynamic turbulence where the $\Delta$-variance is dominated by large scale structures. To detect this effect in star-forming regions observations have to resolve the high density contrast of protostellar cores with respect to their ambient molecular cloud. Using the $3 \mathrm{~mm}$ continuum map of a star-forming cluster in Serpens we show that the dust emission traces the full density evolution. On the contrary, the density range accessible by molecular line observations is insufficient for this analysis. Only dust emission and dust extinction observations are able to to determine the structural parameters of star-forming clouds following the density evolution during the gravitational collapse.
\end{abstract}

Key words. hydrodynamics - ISM: clouds - ISM: magnetic fields - ISM: kinematics and dynamics ISM: structure - turbulence

\section{Introduction}

Stars form through gravitational collapse in clouds of molecular hydrogen. Within these clouds random supersonic gas motions associated with interstellar turbulence lead to transient density fluctuations, some of which may exceed the threshold of gravitational stability and collapse to form stars (e.g. Elmegreen 1993; Padoan 1995; Padoan \& Nordlund 1999).

Detailed studies of the hydrodynamic and magnetohydrodynamic collapse in molecular clouds by Klessen et al. (2000) and Heitsch et al. (2001) show that the location, time scale and efficiency of star formation are intimately connected to the stochastic properties of the underlying turbulent velocity field. To derive a consistent picture of the processes from turbulent fragmentation to star formation it is important to understand how the interplay between supersonic turbulence and self-gravity shapes the density and velocity structure of star-forming

Send offprint requests to: V. Ossenkopf, e-mail: ossk@ph1.uni-koeln.de clouds. Thus we need a quantitative measure for the evolution of the turbulence structure during gravitational collapse. The density and velocity structure have to be compared on different scales to follow the process of structure transformation from the molecular cloud size to the scales of collapsed cores and to understand the influence of gravity on the turbulent driving and the overall cloud structure.

A variety of different statistical measures have been deployed to analyse the structure of turbulence either in observed molecular clouds or in numerical simulations. Many of them are summarised in the reviews by Vázquez-Semadeni (2000) and Ossenkopf et al. (2000). One technique especially sensitive to the discrimination of the relative structural variation on different spatial scales is the $\Delta$-variance introduced by Stutzki et al. (1998). It provides a good separation of noise and observational artifacts from the real cloud structure and for isotropic systems its slope is directly related to the spectral index of the corresponding power spectrum. Bensch et al. (2001) and Mac Low \& Ossenkopf (2000) have shown that it can 
Table 1. Properties of the considered turbulence models together with the resulting time scales.

\begin{tabular}{llcccrr}
\hline model & description & ${k_{\mathrm{d}}}{ }^{2}$ & numerical method & further reference & $\tau_{5 \%}^{c}$ & $f_{\tau_{\mathrm{ff}}}^{d}$ \\
\hline S01 & driven HD turbulence & $1 \ldots 2$ & $\mathrm{SPH}$ & $\mathcal{B} 1 h$ in KHM & 0.6 & $28 \%$ \\
Sd1 & decaying HD turbulence & $1 \ldots 2$ & $\mathrm{SPH}$ & - & 0.6 & $60 \%$ \\
S02 & driven HD turbulence & $7 \ldots 8$ & $\mathrm{SPH}$ & - & $>5.5$ & $0.6 \%$ \\
Sd2 & decaying HD turbulence & $7 \ldots 8$ & $\mathrm{SPH}$ & - & 2.0 & $0.0 \%$ \\
G & Gaussian density & - & $\mathrm{SPH}$ & $\mathcal{I}$ in KB & 1.3 & $9 \%$ \\
H01 & driven HD turbulence & $1 \ldots 2$ & ZEUS & $\mathcal{D} 1 h$ in KHM & 0.6 & $24 \%$ \\
H02 & driven HD turbulence & $7 \ldots 8$ & ZEUS & $\mathcal{D} 3 h$ in KHM & 4.7 & $0.5 \%$ \\
M01 & driven MHD turbulence & $1 \ldots 2$ & ZEUS & $\mathcal{E} h 1 i$ in HMK & 1.2 & $6 \%$ \\
\hline
\end{tabular}

a Wavenumber of the original driving.

${ }^{b}$ Model names in the original papers: KB - Klessen \& Burkert (2000), KHM - Klessen et al. (2000), HMK - Heitsch et al. (2001).

$c$ Time at which $5 \%$ of the total mass is accreted onto cores in internal units where $\tau_{\text {ff }}=1.5$.

${ }^{d}$ Mass fraction that is accreted onto cores after one global free-fall time.

be applied in an equivalent way both to observational data and hydrodynamic and magneto-hydrodynamic turbulence simulations allowing a direct comparison.

Their investigations, however, neglect the influence of gravitational collapse on the structure formation so that their conclusions may be limited when applied to starforming regions. It is essential to include the effects of self-gravity for the analysis of star-forming regions. It is the aim of the current study to close this gap and investigate the interaction between turbulence and self-gravity. We apply the $\Delta$-variance to characterise the structure in numerical models of driven and decaying self-gravitating supersonic (magneto-)hydrodynamic turbulence and compare the results to observed regions of star formation.

In Sect. 2 we introduce the molecular cloud models analysed here and the numerical methods used for their generation. In Sect. 3 we apply the $\Delta$-variance analysis and discuss the time evolution of the structure as local collapse sets in and protostellar cores form and accrete mass. Using various models and comparing the density and velocity structure we demonstrate how power is build up at the different scales during star formation. Section 4 provides a comparison with observational data including dust emission and molecular lines and discusses the resulting differences. We summarise our results in Sect. 5 .

\section{Turbulence models}

The large observed linewidths in molecular clouds imply the presence of supersonic velocity fields that carry enough energy to counterbalance gravity on global scales (Williams et al. 2000). As turbulent energy dissipates rapidly, i.e. roughly on the free-fall time scale (Mac Low et al. 1998; Stone et al. 1998; Padoan \& Nordlund 1999), either interstellar turbulence is continuously replenished in order to prevent or considerably postpone global collapse, or alternatively, molecular clouds evolve rapidely and never reach dynamical equilibrium between kinetic energy and self-gravity (Ballesteros-Paredes et al. 1999a; Elmegreen 2000). Supersonic turbulence may be driven by Galactic rotation and supernovae on large scales and by outflows from young stellar objects on small scales (see e.g. de Avillez 2000). The current models investigate both cases, continuously replenished and freely decaying turbulence.

We select a set of numerical models mostly from preexisiting studies that spans a large range of the parameter space relevant for Galactic molecular clouds. We analyse the time evolution of their density and velocity structure as gravitational collapse progresses. Altogether, we include eight models summarised in Table 1 . They differ in the scale on which turbulent driving occurs, the persistence of this driving, the inclusion of magnetic fields, and the numerical algorithm used to solve the hydrodynamic or magnetohydrodynamic equations.

In the present analysis we neglect feedback effects from the produced young stellar objects (like bipolar outflows, stellar winds, or ionising radiation from new-born $\mathrm{O}$ or $\mathrm{B}$ stars). Thus our results will hold only for early stages of star-forming systems.

\subsection{The driving process}

To represent hydrodynamic turbulence which carries most energy on large scales we consider a freely decaying (model Sd1) and a driven case (S01). Turbulence that is driven at large wavelength leads to a clustered mode of star formation (Klessen et al. 2000) and appears to yield the most appropriate description of molecular cloud dynamics (see e.g. Ossenkopf \& Mac Low 2001). Furthermore, we consider decaying and driven small-scale hydrodynamic turbulence (models Sd2 and S02, respectively).

In all driven models the energy input is adjusted to yield the same turbulent Mach number $\mathcal{M}_{\mathrm{rms}}=$ 10. The Mach number is calculated from the threedimensional $\mathrm{rms}$ velocity dispersion, $\mathcal{M}_{\mathrm{rms}} \equiv v_{\mathrm{rms}} / c_{\mathrm{s}}=$ $\sqrt{2 E_{\text {kin }} / M} / c_{\mathrm{s}}$, where $c_{\mathrm{s}}$ is the sound speed, $E_{\text {kin }}$ the total kinetic energy and $M$ the total mass in the system. Turbulent equilibrium is obtained applying a non-local 
driving scheme, that inserts energy in a limited range of wavenumbers such that the total kinetic energy contained in the system remains constant and compensates the gravitational contraction on global scales (Mac Low 1999).

For the decaying models this Mach number holds at times $t \leq 0$, before turbulence is allowed to decay. The driving stops when gravity is "turned on" at $t=0$ when the stage of fully established turbulence is reached. We also consider a case of large-scale contraction from Gaussian density fluctuations without any turbulent support (model G). This represents the most extreme case of clustered star formation (Klessen 2001).

\subsection{The numerical implementation}

All models are computed either using SPH (smoothed particle hydrodynamics), a particle-based Lagrangian scheme to solve the equations of hydrodynamics, or ZEUS, a gridbased code. We directly compare the two codes in the case of driven turbulence. Here we accompany the SPH models S01 and S02 by the corresponding ZEUS models H01 and H02. This has proven to be a very effective means of distinguishing physical results from numerical artifacts (Mac Low et al. 1998), as these two approaches practically bracket the real dynamical behaviour of interstellar turbulence. The density enhancements are spatially somewhat too small and rigid in the SPH model and too extended in the grid-based compuations (discussed in detail by Klessen et al. 2000).

In $\mathrm{SPH}$ the fluid is represented by an ensemble of particles, where thermodynamical observables are obtained as local averages (Benz 1990; Monaghan 1992). The method is very flexible and can resolve high density contrasts by increasing the particle concentration where needed. We use SPH in combination with the special-purpose hardware device GRAPE (Sugimoto et al. 1990; Ebisuzaki et al. 1993), which allows calculations at supercomputer level on a normal workstation, and adopt periodic boundary conditions (Klessen 1997) focusing on the evolution of a sub-region within a larger, globally stable molecular cloud.

The simulations use $2 \times 10^{5} \mathrm{SPH}$ particles for all turbulence models except the collapse of the Gaussian density fluctuations where we use $5 \times 10^{5} \mathrm{SPH}$ particles. Shock-generated density fluctuations with masses exceeding the local Jeans limit become unstable and collapse. Once their central nuclei exceed a density contrast of $10^{5}$, they are identified as protostellar cores and replaced by "sink" particles (Bate et al. 1995). These inherit the combined masses, linear and "spin" angular momenta of the replaced particles, and have the ability to accrete further $\mathrm{SPH}$ particles from their infalling gaseous envelopes. This technique allows us to follow the dynamical evolution of turbulent molecular cloud regions over many global freefall time scales. The numerical scheme and the scaling properties of the model are discussed in detail by Klessen \& Burkert (2000) and Klessen et al. (2000).
For the grid-based simulations we use the astrophysical MHD code ZEUS-3D (Clarke 1994). This is a threedimensional version of the code described by Stone \& Norman (1992a,b) using second-order advection (van Leer 1977) and von Neumann artificial viscosity introduced to resolve shocks. Moreover, structures close to the grid resolution are subject to numerical dissipation as discussed by Mac Low \& Ossenkopf (2000).

The simulations were performed on a threedimensional, uniform, Cartesian grid containing $256^{3}$ cells and assuming periodic boundary conditions. All quantities are scaled to match the corresponding definitions in the SPH simulations (see also Heitsch et al. 2001).

\subsection{Magnetic fields}

To test the influence of magnetic fields we consider the driven turbulence model which carries most energy on large scales and add magnetic fields to the ZEUS simulations. By comparing the resulting model M01 with the hydrodynamic model $\mathrm{H} 01$ we get a direct measure for the significance of magnetic fields for the interplay of turbulence and self-gravity in structure formation.

The magnetic field in this model is selected to be a major factor where the ratio between thermal and magnetic pressure $\beta=p_{\text {th }} / p_{\text {mag }}=8 \pi c_{\mathrm{s}}^{2} \rho / B^{2}=0.04$. With a turbulent Mach number of $\mathcal{M}_{\mathrm{rms}}=10$ we find that the turbulent velocity dispersion exceeds the Alfvén speed by a factor of 1.4 so that the structure is still essentially determined by supersonic turbulence and only modified by the magnetic field. The mass in the cloud still exceeds the critical mass for a magnetostatically stable cloud by a factor 2 (Heitsch et al. 2001) so that the field should not prevent gravitational collapse. Cases of sub-Alfvénic non-self-gravitating turbulence where the whole structure is dominated by the magnetic field have been discussed by Ossenkopf \& Mac Low (2001).

\subsection{Scaling}

The models presented here are computed in normalised units. Throughout this paper we give all size values relative to the total cube size, all density values relative to the the average density in the cube, and all velocities relative to the sound speed. Model G contains 220 thermal Jeans masses, wherease all other models have 120 thermal Jeans masses ${ }^{1}$. If scaled to mean densities $n\left(\mathrm{H}_{2}\right)=$ $10^{5} \mathrm{~cm}^{-3}$, a value typical for star-forming molecular cloud regions and a temperature of $11.4 \mathrm{~K}$ (i.e. a sound speed $\left.c_{\mathrm{s}}=0.2 \mathrm{~km} \mathrm{~s}^{-1}\right)$, the mean Jeans mass is one solar mass,

\footnotetext{
${ }^{1}$ Throughout this paper the spherical definition of the Jeans mass is used, $M_{\mathrm{J}} \equiv 4 / 3 \pi \rho \lambda_{\mathrm{J}}^{3}$, with density $\rho$ and Jeans length $\lambda_{\mathrm{J}} \equiv(\pi \mathcal{R} T / G \rho)^{1 / 2}$, where $G$ and $\mathcal{R}$ are the gravitational and the gas constant. The mean Jeans mass $\left\langle M_{\mathrm{J}}\right\rangle$ is determined from average density in the system $\langle\rho\rangle$. An alternative cubic definition, $M_{\mathrm{J}} \equiv \rho\left(2 \lambda_{\mathrm{J}}\right)^{3}$, would yield a value roughly twice as large.
} 
$\left\langle M_{\mathrm{J}}\right\rangle=1 M_{\odot}$, and the size of cube $\mathrm{G}$ is $0.34 \mathrm{pc}$ whereas all other models have a size of $0.29 \mathrm{pc}$. The global freefall time scale, as defined by $\tau_{\mathrm{ff}}=(3 \pi / 32 G)^{1 / 2}\langle\rho\rangle^{-1 / 2}$ with $\langle\rho\rangle$ being the average density, is about $10^{5} \mathrm{yr}$. In normalised time units it follows $\tau_{\mathrm{ff}}=1.5$. The simulations cover a density range from $n\left(\mathrm{H}_{2}\right) \approx 100 \mathrm{~cm}^{-3}$ in the lowest density regions to $n\left(\mathrm{H}_{2}\right) \approx 10^{9} \mathrm{~cm}^{-3}$ where collapsing protostellar cores are identified and converted into "sink" particles in the SPH code.

In this density regime gas cools very efficiently and it is possible to use an effective polytropic equation-ofstate in the simulations instead of solving the detailed equations of radiative transfer. The effective polytropic index is typically close to unity, $\gamma_{\text {eff }} \lesssim 1$, except for densities $10^{5} \mathrm{~cm}^{-3}<n\left(\mathrm{H}_{2}\right)<10^{7} \mathrm{~cm}^{-3}$, where somewhat smaller values of $\gamma_{\text {eff }}$ are expected (Spaans \& Silk 2000). For simplicity, we adopt a value of $\gamma_{\text {eff }}=1$, i.e. an isothermal equation of state for all densities in the simulations. As the choice of $\gamma_{\text {eff }}$ influences the density contrast in shock compressed gas, this idealisation may introduce some small modifications to the dynamical behaviour compared to real cloud systems (see Scalo et al. 1998 or Ballesteros-Paredes et al. 1999b for further discussion).

\subsection{Model results}

Supersonic turbulence, even if strong enough to counterbalance gravity on global scales will usually provoke local collapse. Turbulence establishes a complex network of interacting shocks, where converging shock fronts generate clumps of high density. This density enhancement can be large enough for the fluctuations to become gravitationally unstable and collapse. Long-wavelength turbulence is dominated by large-scale shocks which are very efficient in sweeping up molecular cloud material, thus creating massive coherent structures. When a coherent region reaches the critical density for gravitational collapse its mass typically exceeds the local Jeans limit by far. Inside the shock compressed region, the velocity dispersion is much smaller than in the ambient turbulent flow and the situation is similar to localised turbulent decay. Quickly a cluster of protostellar cores forms. Therefore, all our models with zero support, decaying, or large-scale driven turbulence, similarly lead to a clustered mode of star formation. The efficiency of turbulent fragmentation is reduced if the driving wavelength decreases. When energy is inserted mainly on small spatial scales, the network of interacting shocks is very tightly knit, and protostellar cores form independently of each other at random locations throughout the cloud and at random times with low efficiency corresponding to an isolated mode of star formation (Klessen et al. 2000, Fig. 1 in Klessen 2001). The effect of magnetic fields strongly depends on their strength. Whereas for the subcritical case, i.e. for fields which can support a gravitationally unstable region magnetostatically (Mouschovias \& Spitzer 1976) no collapse occurs, supercritical weak fields reduce the core growth rate perceptibly, although they cannot prevent local collapse (Heitsch et al. 2001).

To quantify the above we indicate in Table 1 the time when local collapse has accumulated $5 \%$ of the total mass in dense cores and also give the core mass fraction after one free-fall time scale. Hence, we can directly compare the $\Delta$-variance and other the structural properties of the different models either at equal times or at equivalent degrees of collapse.

\section{The evolution of structural properties}

\subsection{The $\Delta$-variance analysis}

The $\Delta$-variance analysis was introduced by Stutzki et al. (1998) as an averaged wavelet transform (Zielinsky \& Stutzki 1999) to measure the amount of structure present at different scales in an $E$-dimensional data set. The value of the $\Delta$-variance at a certain scale is computed by convolving the $E$-dimensional structure with a normalised spherically symmetric down-up-down function of the considered size, and measuring the remaining variance. For two-dimensional structures, like astrophysical maps, the filter function is easily visualized as a "French hat" wavelet with a positive centre surrounded by a negative ring and equal diameters of the centre and the annulus. The analysis can be applied in the same way in arbitrary dimensions by extending the filter function to higher dimensions retaining its radial symmetry and adapting the value in the negative part to preserve normalization (Appendix B of Stutzki et al. 1998). The resulting $\Delta$-variance as a function of the filter size measures the amount of structural variation on that scale.

As the convolution with the filter function corresponds to a multiplication in Fourier space, we can relate the $\Delta$-variance to the power spectrum of the data set. If the structure is characterised by a power law power spectrum $P(|\boldsymbol{k}|) \propto|\boldsymbol{k}|^{-\beta}$

the slope $\alpha$ of the $\Delta$-variance as a function of lag (filter size) is related to the spectral index $\beta$ of the power spectrum by $\beta=\alpha+E$ for $0 \leq \beta<E+4$. Due to the smooth circular filter function the $\Delta$-variance measures the spectral index in a way which is more robust with respect to edge and gridding effects than the Fourier transform. The $\Delta$-variance provides a clear spatial separation of different effects influencing observed structures like noise or a finite observational resolution.

Mac Low \& Ossenkopf (2000) have shown that we can translate the $\Delta$-variance of a three-dimensional isotropic structure into the $\Delta$-variance of the maps obtained from the projections of this structure by rescaling with a factor proportional to the lag and an additional small shift. This guarantees the preservation of the power spectral index $\beta$ in projection (Stutzki et al. 1998). As we want to compare the simulations to observational data we will always use the two-dimensional representation of the $\Delta$-variance results also when applying the analysis directly to the threedimensional data cubes of the simulations. To compute 


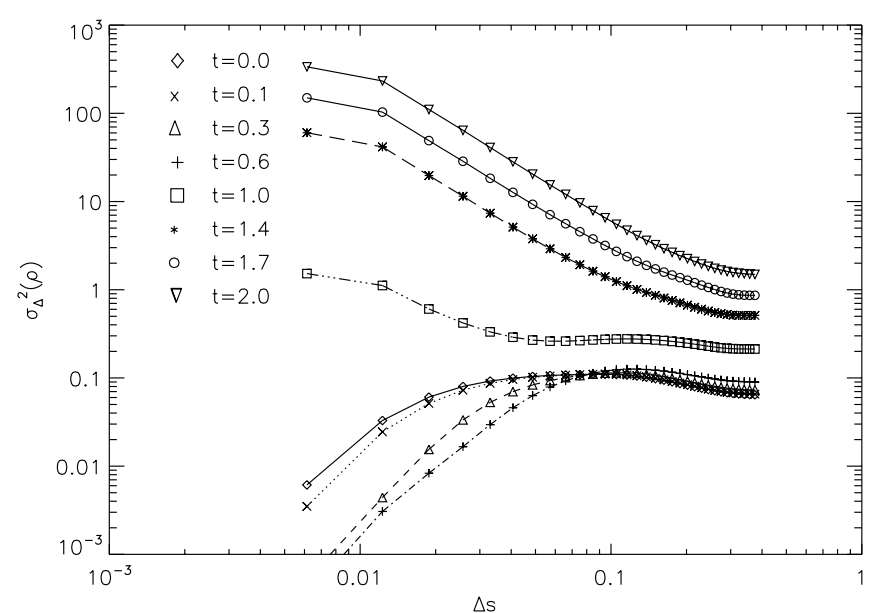

Fig. 1. Time evolution of the strength of density fluctuations as function of their spatial scale measured with the $\Delta$-variance for model G. The density $\rho$ is given in units of the average density in the cube, the lag $\Delta s$ in units of the cube size, and the time $t$ in internal time units where the free-fall time $\tau_{\mathrm{ff}}=1.5$.

the $\Delta$-variance for our models the SPH density distribution is assigned onto a Cartesian grid with $128^{3}$ cells. The ZEUS cubes have been analysed in full resolution at $256^{3}$ as well as degraded to $128^{3}$ for a one-to-one comparison with the SPH models. Higher resolution helps to extend the dynamic scale range limited by the periodic boundary conditions at the large scale end and the numerical resolution of the simulations on the small scale end, but does not change the general behaviour of the resulting $\Delta$-variance.

\subsection{The evolution of the density structure}

\subsubsection{The collapse of a Gaussian density field}

Before we investigate the interplay between supersonic turbulence and self-gravity, let us consider a system where the density and velocity structure is dominated by gravity on all scales and at all times. Model G describes the collapse of Gaussian density fluctuations with initial power spectrum $P(k) \propto k^{-2}$ and maximum density contrast $\delta \rho / \rho \approx 50$. The system is unstable against gravitational collapse on all scales and forms a cluster of protostellar cores within about two free-fall time scales (Klessen \& Burkert 2000, 2001). The velocity structure is coupled to the density distribution via Poisson's equation and there is no contribution from interstellar turbulence.

The time evolution of the density structure is illustrated in Fig. 1. Initially, the $\Delta$-variance $\sigma_{\Delta}^{2}(n)$ is more or less constant $(\alpha=0)$ on scales $\Delta s \gtrsim 0.02$, in agreement with the initial power spectrum $P(k) \propto k^{-2}$. The steepening below $\Delta s \approx 0.02$ is produced by the finite resolution of the $\mathrm{SPH}$ simulations resulting in the blurring of structures at the smallest scales.

The first changes of the variance $\sigma_{\Delta}^{2}(n)$ are confined to small scales. Initial fluctuations with masses below the local Jeans limit will quickly smear out by thermal pressure as the system evolves from purely Gaussian fluctuations into a hydrodynamically self-consistent state (see Appendix B in Klessen \& Burkert 2000). As these fluctuations are by far more numerous than Jean-unstable contracting ones, the $\Delta$-variance $\sigma_{\Delta}^{2}(n)$ begins to decrease on small scales. However, as the central regions of massive Jeans-unstable fluctuations contract to sufficiently high densities, $\sigma_{\Delta}^{2}(n)$ increases again. This mainly affects the small scales as local collapse modifies the density structure on time scales of the local free-fall time. At $t=0.7 \tau_{\mathrm{ff}}$ the first collapsed core is identified and is soon followed by others. Altogether 56 dense protostellar cores build up. As time advances larger and larger scales exhibit noticeable signs of contraction. After about one global free-fall time collapse starts to involve all spatial modes in the system and the absolute magnitude of the density fluctuations finally grows on all scales. As the small scale structure dominates the density structure we obtain a negative slope in the $\Delta$-variance spectrum. In the final step of the simulation roughly $30 \%$ of the mass is accumulated in dense cores and the slope is about -1.7 indicating that a small but significant contribution of large scale structure is still present, because an uncorrelated $N$-body system of gravitationally collapsed points would correspond to a slope of -2 equivalent to a flat power spectrum $P(k)=$ const.

The flattening at $\Delta s>0.2$ is due to periodicity. The system is not allowed to collapse freely, it is held up against global collapse by periodic boundaries which strongly affect the evolution of the large-scale modes. The graphs of $\Delta$-variance are not extended beyond effective lags of about 0.4 as the largest filter that we use is half the cube size and we have to apply an average length reduction factor of $\pi / 4$ on projection to two dimensions.

\subsubsection{The interaction between gravitation and turbulence}

To study the interplay between supersonic turbulence and self-gravity, we consider four models of interstellar turbulence which probe very disparate regions of the relevant parameter space. In models S01 and Sd1 most of the turbulent kinetic energy is carried on large scales, whereas models S02 and Sd2 involve mainly small-scale turbulent modes. In S01 and S02, turbulence is continuously driven such that at any moment the overall kinetic energy compensates the global gravitational energy. In $\mathrm{Sd} 1$ and $\mathrm{Sd} 2$, the turbulent energy is allowed to decay freely.

Figure 2 shows $\sigma_{\Delta}^{2}(n)$ for all four models as function of time. In the initial plots one can clearly see the dominance of the driving scale as discussed by Mac Low \& Ossenkopf (2000). The introduction of a velocity field with a certain scale induces a pronounced peak in the density structure at a somewhat smaller scale. Thus the curves at $t=0$ show for the large scale-driven models a power law $\Delta$-variance from about a third of the cube size down to the numerical dissipation scale whereas in the small-scale driven model the driving feature at about 0.07 dominates the structure. 

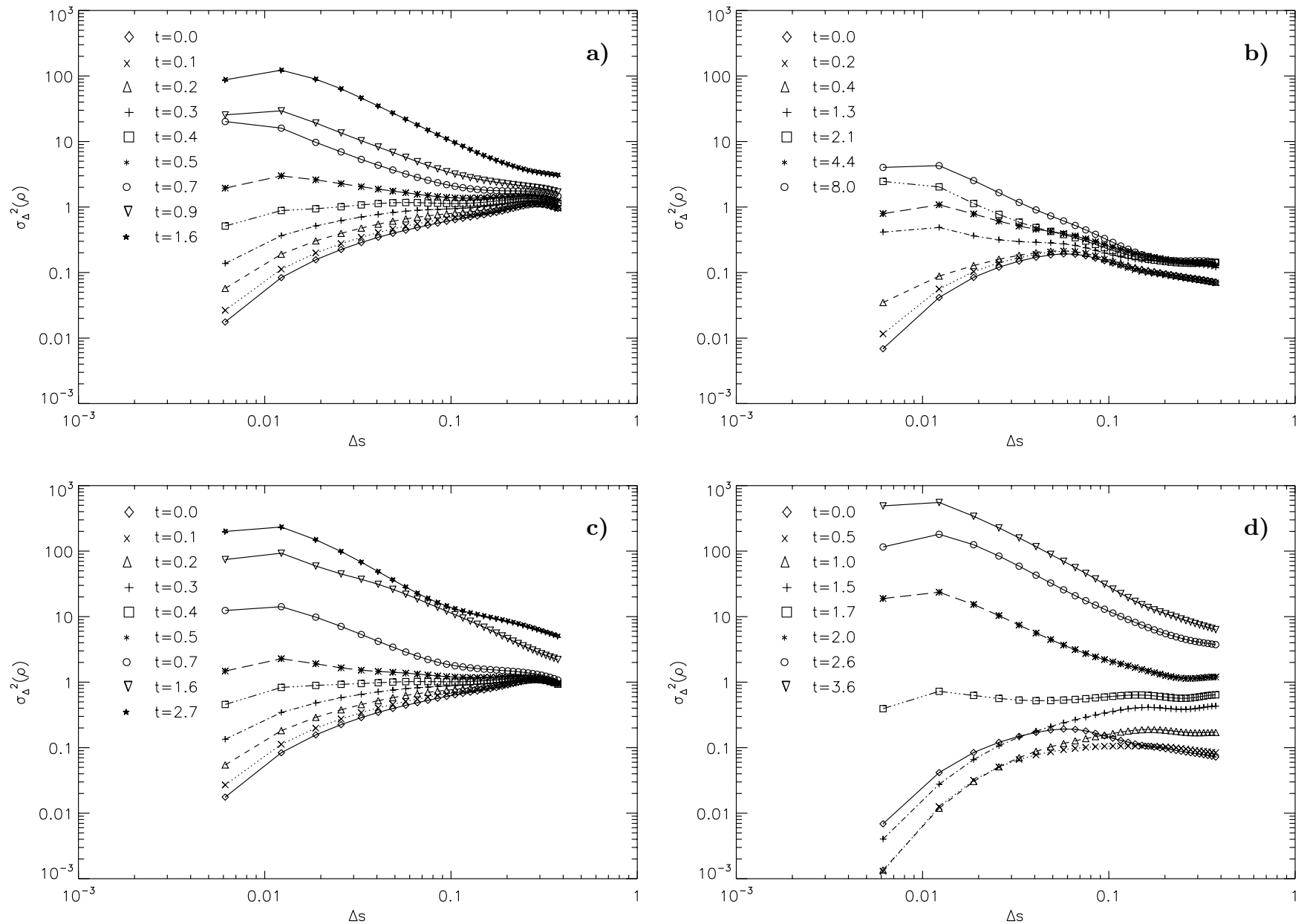

Fig. 2. Time evolution of the size distribution of density variations as measured with the $\Delta$-variance for the four models of a) $\mathrm{S} 01, \mathbf{b}) \mathrm{S} 02$, c) $\mathrm{Sd} 1$, and d) $\mathrm{Sd} 2$. The different depicted times are indicated at the left side of each plot.

Star formation is a joint feature of all considered models. Like in the evolution of the Gaussian density field the gravitational collapse first modifies only the smallest scales, hardly changing the global behaviour. As soon as local collapse occurs and the first dense protostellar cores form and grow in mass by accretion, they represent the main density fluctuations. Their power is concentrated on small scales and the $\Delta$-variance exhibits a negative slope. The structure resulting from the collapse is very similar in the various models when we compare evolutionary stages with about the same mass fraction collapsed onto cores.

In the initially large-scale driven models a turbulent cascade covering all scales is already present from the beginning shown by the highest $\sigma_{\Delta}^{2}(n)$-values at long scales. Within this cascade the number of small-scale fluctuations is small compared to models S02, Sd2, and G, and they are typically part of a larger structure so that they are only weakly dispersed in time. This leads to a monotonous growth of the $\Delta$-variance on all scales. As larger and larger regions become gravitationally unstable the contraction comprises increasingly larger scales, and finally $\sigma_{\Delta}^{2}(n)$ shifts "upward" on all scales while maintaining a fixed slope. This situation is similar in all models with allow for large-scale collapse, i.e. it is only prevented in the small scale-driven model S02.

A different temporal behaviour is visible in $\mathrm{Sd} 2$, the decaying turbulence which was originally driven at small scales. Like in the collapse of the Gaussian density field we find in the first steps of the gravitational evolution a relative reduction of small-scale structure. This decrease is due to the termination of the initial small-scale driving resulting in a quick dissipation of the existing fluctuations by thermal pressure if they are not Jeans supercritical, analogously to the Gaussian collapse case. In the next steps we notice the production of structure on larger scales. The smoothing of small-scale turbulence combined with the onset of self-gravity leads to global streaming motions which produce density structures correlated on a larger scale. Large-scale structures had been initially suppressed by the non-local turbulent driving mechanism. After less than one free-fall time a kind of self-sustaining inertial cascade with a $\Delta$-variance slope $\alpha$ of about 0.5 is build up like in all other decaying models and in the large scale driven model. After these initial adjustments the first protostellar cores form and we find the same dynamical behaviour. 
The time scale to reach a comparable collapse state and the final structure that we reach in the simulations differs between the models, mainly determined by the strength of the turbulent driving. The exponent $\alpha$ of the $\Delta$-variance in the collapsed state is -1.3 in the models containing continuous driving, -1.5 in the models where the turbulence decays during the gravitational collapse, and -1.7 for the pure collapse of the Gaussian density field. This is understandable, as decreasing turbulent support leads to enhanced collapse forming stars in denser clusters. The final deviation from the uncorrelated field of protostars which has $\alpha=-2$ is thus a measurable indicator of the turbulent processes in the cloud during gravitational collapse. The local collapse produces "point-like" high-density cores with small overall filling factor whereas most of the volume is supported by hydrodynamic turbulence. In the large-scale driven model $30 \%$ of the mass and in the decaying models $60 \%$ of the total mass has turned into cores within the considered time interval. In the model continuously driven at small scales only $3 \%$ of the mass is in stable cores despite a considerably longer simulation time. The small-scale driven turbulence leads to the least efficient star formation in an isolated mode, whereas the other cases result in the formation of stellar aggregates and clusters (see also Klessen et al. 2000; Klessen 2001).

Beside the deceleration of collapse the continuation of the initial driving has almost no influence on the resulting density structure as soon as the first stable cores have formed. It only maintains a constant level of velocity fluctuations in the main volume of the cloud which is dominated by low density gas, compared to a homogeneous reduction of these fluctuations in the purely decaying case.

\subsubsection{The influence of the numerical model}

Comparing the results of the particle-based SPH and the grid-based ZEUS code we can distinguish between numerical artifacts and physical results, as these two approaches practically bracket the real dynamical behaviour of interstellar turbulence. In Fig. 3 we compare the $\Delta$-variance plots of the density structure obtained for the driven cases using either SPH or ZEUS, at the beginning of the gravitational collapse and in a step where the structure is already dominated by protostellar cores.

The scaling behaviour of the density structure does not differ between both types of simulations but the absolute magnitude of the density fluctuations as seen in the total value of the $\Delta$-variance is somewhat larger for all ZEUS models. In the first steps of the large-scale driven models both numerical approaches still agree approximately but during the evolution the scale dependent density variations become about a factor five higher in the ZEUS model than in the SPH approach. In the small scale driven models we can notice a clear difference already at the beginning of the simulations. This is consistent with the different effective resolution of the methods. Whereas the $\mathrm{SPH}$
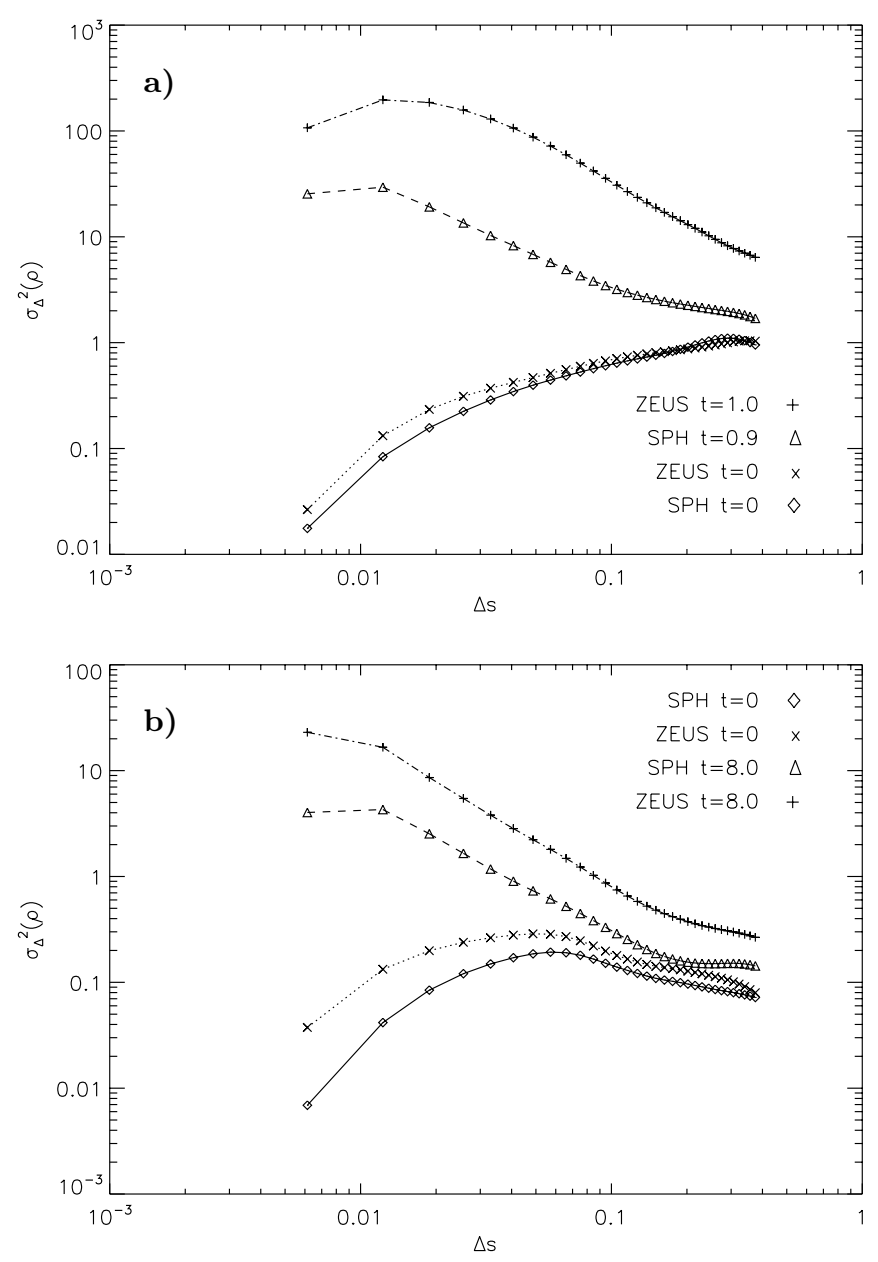

Fig. 3. Comparison of the $\Delta$-variance measured from the particle-based and the grid-based simulations at the beginning and at about the same timestep of the gravitationally collapsed state. Plot a) shows the large-scale driven model, plot b) the small scale-driven situation.

code can provide a very good spatial resolution around the collapsing dense regions the general spatial resolution obtained with $2 \times 10^{5}$ particles is lower than in the ZEUS simulations on a $256^{3}$ grid. Thus, the damping of structures at small scales due to the finite resolution of the code is slightly stronger in the SPH simulations than in the corresponding ZEUS models. One can for instance see that there is a virtual reduction of structure below 0.01 which is approximately the radius of the sink particles in the SPH code.

As the SPH resolution is explicitly density dependent it is also reduced on all larger scales in low density regions. This virtually smears out part of the structure on all scales. Consequently, the $\Delta$-variance shows lower values on all scales than in the grid-based approach because it is not biased towards high-density regions like SPH and the observations. The effect is larger in the small-scale driven models as the same number of SPH particles has to represent more shocks than in large-scale dominated cases further reducing the effective resolution. Moreover, the resolution worsens during the collapse evolution as 


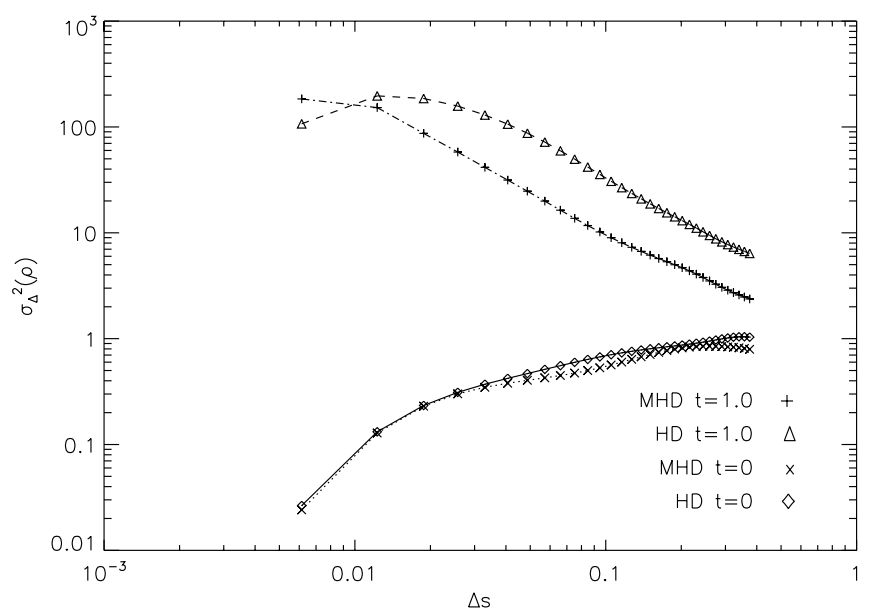

Fig. 4. Comparison of the $\Delta$-variance for the large-scale driven model in the hydrodynamic case or the situation with strong magnetic fields, both computed with the ZEUS code.

SPH particles "vanish" in the sink particles. Thus, the ZEUS simulations are preferential due to their higher resolution if one is interested in the absolute value of the $\Delta$-variance whereas they provide no essential advantage for the study of the scaling behaviour.

\subsubsection{Magnetic fields}

As discussed by Ossenkopf \& Mac Low (2001) magnetic fields hardly change the general scaling behaviour in interstellar turbulence but create anisotropies in the velocity field and therefore aligned density structures. Since the $\Delta$-variance cannot measure anisotropies in the density structure we do not expect to detect the influence of the magnetic field on the turbulence by the present analysis. Figure 4 shows the $\Delta$-variance for the initial step and an collapsed stage in a large-scale driven hydrodynamic model and the equivalent MHD model with a strong magnetic field. The initial steps are almost identical but we find that during collapse the magnetic field effectively helps to transfer structure from larger to smaller scales. Thus we confirm the more qualitative conclusion of Heitsch et al. (2001) that the magnetic field slightly delays collapse by transferring part of the turbulent kinetic energy to smaller scales. The general slope of the $\Delta$-variance is not changed but we obtain somewhat denser and smaller cores and somewhat less large-scale correlation at equivalent timesteps.

Computing the $\Delta$-variance for maps projected either in the direction of the initial magnetic field or perpendicular to it does not show any significant difference in the density scaling behaviour as mainly the shape of the collapsed regions is influenced, towards spiral-shaped structures, which is not measurable with the isotropic $\Delta$-variance filter.

We have also tested models with a smaller magnetic field where the magnetic pressure in the order of the thermal pressure or lower. Here, we find that the field acts like

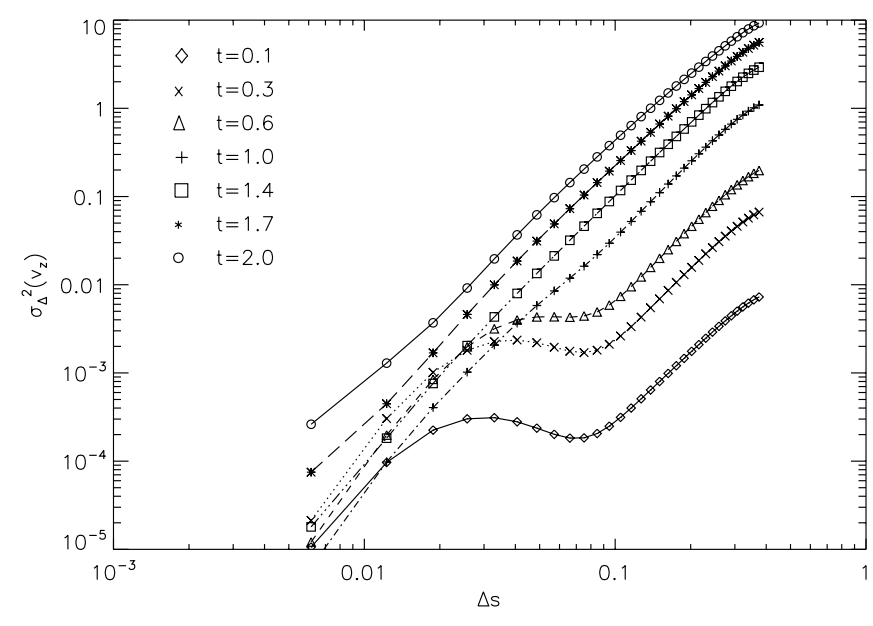

Fig. 5. Evolution of the $\Delta$-variance of the $z$-velocity in the collapse of the Gaussian density distribution (model G). The velocity $v_{z}$ is given here in units of the thermal sound speed $c_{\mathrm{s}}$.

an additional contribution to the overall isotropic pressure so that the collapse is somewhat delayed relative to the hydrodynamic case but the general structure does not deviate from the hydrodynamic simulations. Thus there is no need to discuss the weak-field situation here separately.

\subsection{The velocity structure}

We can apply the $\Delta$-variance analysis in the same way to the velocity structure in the simulations (Ossenkopf \& Mac Low 2001). Figure 5 shows the evolution of one velocity component in the collapse of the Gaussian density fluctuations. In the first steps where we observe a relative reduction of small scale density fluctuations we find a bimodal velocity distribution with either very small or very large flows. The surplus of small-scale flows just reflects the dissipation of the initial small-scale variations by thermal pressure. When the first stable cores have formed the picture changes towards that of a typical shock-dominated medium with a slope $\alpha=2$ (Ossenkopf \& Mac Low 2001) as the result of supersonic accretion onto dense cores along the emerging filamentary structure (Klessen \& Burkert 2000, 2001).

In all driven models we see no significant changes in the velocity structure during collapse. This is because the $\Delta$-variance is not focused towards the dense cores where collapse motions occur, as these have only a small spatial filling factor. Instead, most of the volume is occupied by tenuous intercore gas with velocity structure that is determined by turbulent driving. The $\Delta$-variance therefore exhibits the power-law behavior of shock dominated gas.

The same hold for decaying turbulence as well. To illustrate that point, Fig. 6 presents the evolution of the velocity structure for model Sd1, where we drive the turbulence initially at large scales and switch off the driving during the gravitational collapse. The changes in the velocity structure are only minute. The $\Delta$-variance follows the power law of shock-dominated flows throughout the 


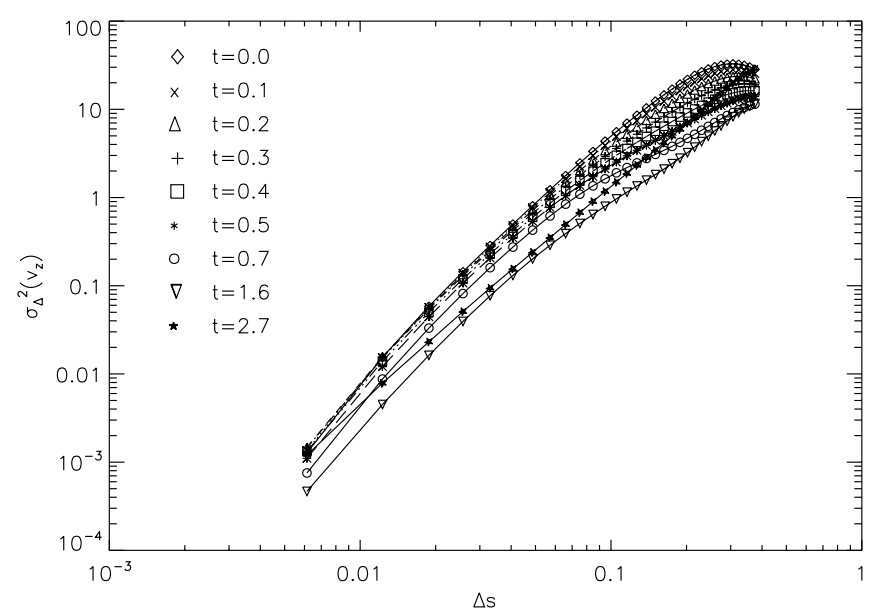

Fig. 6. Evolution of the $\Delta$-variance of the $z$-velocity component of the decaying model Sd1.

entire evolution. Only the total magnitude of the velocity fluctuations decreases slightly during the initial decay of turbulence. However, after the onset of collapse, when the majority of mass is already accumulated in dense cores, the magnitude of $\sigma_{\Delta}$ increases again. The $\Delta$-variance becomes dominated by the shock structure arising from the supersonic accretion flows onto individual cores (similar to the late stages of model G). The evolution of the smallscale decaying turbulence is not plotted separately, as we observe the same behavior. Except during the initial phase of turbulent decay where the velocity structure still peaks on small scales reflecting the smaller driving wavelength used to set up the model (see Fig. $3 \mathrm{~d}$ for the density structure), again after $t \approx 1.5$ when the initial turbulence is sufficiently decayed away the $\Delta$-variance arrives at the power-law behavior of shocked gas.

As a point of caution, we note here that the velocity structure of all SPH models exhibits a numerical artifact due to the density weighted selection of the size used for the averaging kernel. This produces virtual velocity streams with relatively large coherence length at low densities resulting in a $\Delta$-variance slope $\alpha$ of about 2.8 significantly above the value of 2 characteristic for a shockdominated medium. As the weighting function is given by the density of SPH particles, this effect is only visible in the velocity structure and not in the density scaling. The corresponding grid-based models do not exhibit a similar enhancement of large scales in the velocity structure and show a $\Delta$-variance slope of about 2 .

In Fig. 7 we show the three velocity components of the MHD model M01 at the same two timesteps like in the density plot in Fig. 4. In contrast to the findings of Ossenkopf \& Mac Low (2001) for sub-Alfvénic turbulence, we see no strong anisotropy of the velocity field. The velocity structure along the mean magnetic field ( $z$-direction) is very similar to the perpendicular directions throughout the dynamical evolution of the system and well within the statistical fluctuations expected for large-scale turbulence. The velocity structure is still

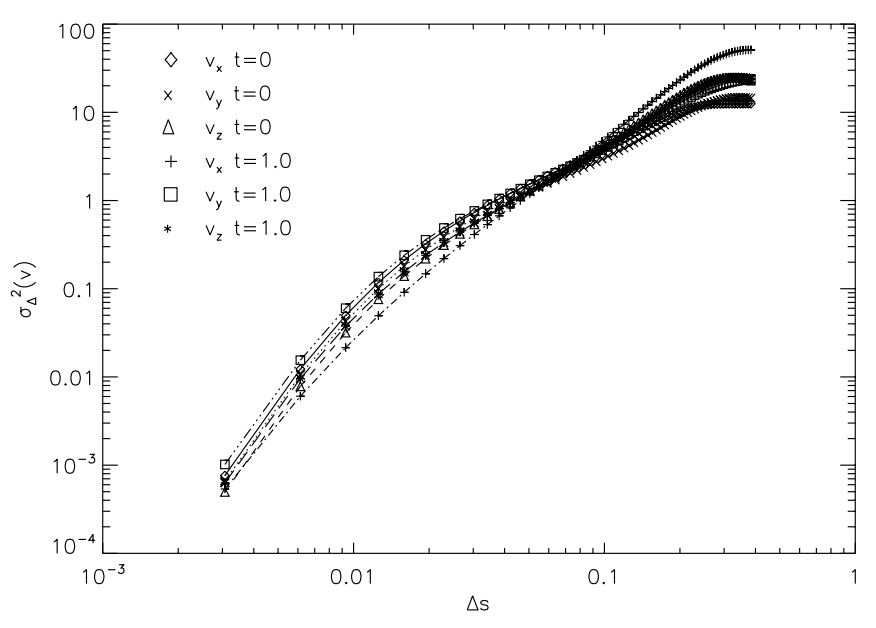

Fig. 7. $\Delta$-variance of all three velocity components for model the large-scale driven MHD model at the initial step and after one free-falling time.

determined by supersonic turbulence rather than by the magnetic field structure because the turbulent rms velocity dispersion exceeds the Alfvén speed in this model. The local collapse and the formation of a cluster of collapsed cores tends to make the influence of the magnetic field on the velocity structure even weaker. The magnetic field merely decelerates the gravitational collapse and changes the geometry of the collapsing regions as seen in Fig. 4, but hardly changes the global velocity structure in this model situation.

Altogether we find that all models that are allowed to evolve freely or are driven at large scales exhibit a similar velocity scaling behaviour, characteristic of shockdominated media. This is the effect of the undisturbed turbulence evolution and the appearance of accretion shocks. Both effects lead to remarkably similar properties of the velocity $\Delta$-variance. Any observed deviation from this large-range "Larson" behaviour indicative of shockdominated media will hint the presence of additional physical phenomena and could provide constraints on the initial conditions and the dynamical state of star-forming regions.

\section{Comparison with observations}

\subsection{Dust observations}

To compare the simulations of gravitational collapse with observational data of collapsed regions we have selected the observation of a star-forming cluster in Serpens by Testi \& Sargent (1998) as this region represents a state of star formation that may be similar to the outcome of our simulations.

In Fig. 8 we show the $\Delta$-variance for the $3 \mathrm{~mm}$ dust continuum map of Testi \& Sargent (1998). It reveals an increase of the relative amount of structure from small scales towards a peak at $7^{\prime \prime}$, an intermediate range which can be fitted by a power-law exponent $\alpha=-1.2$, and a decay with $\alpha=-2$ indicating the complete lack of 


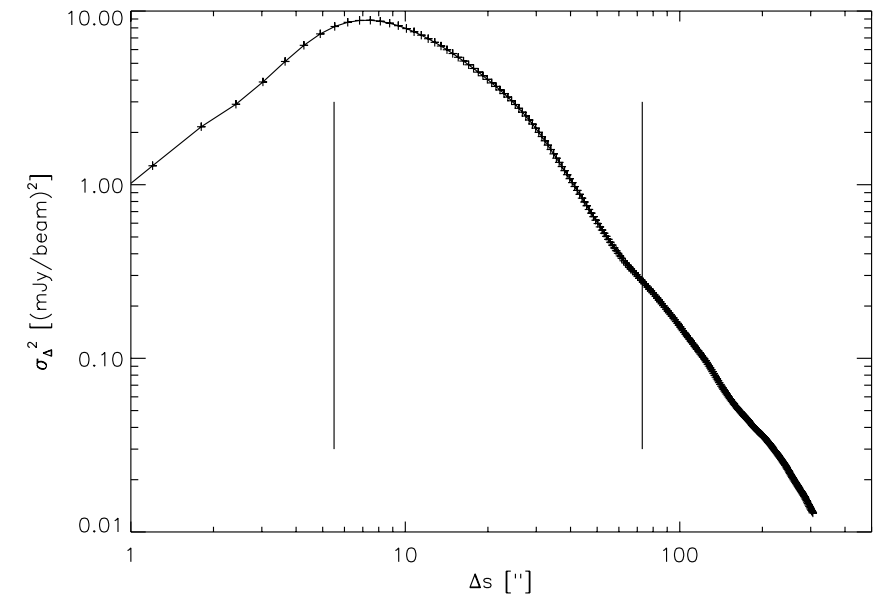

Fig. 8. $\Delta$-variance of the dust continuum map in Serpens taken by Testi \& Sargent (1998). The two vertical lines represent the limits of the significant range as indicated with the observational data.

large-scale structure at lags above $40^{\prime \prime}$. The behaviour at largest and smallest scales can be understood when looking at the observational base of the map. Testi \& Sargent (1998) give a resolution for their interferometric observations of $5.5^{\prime \prime} \times 4.3^{\prime \prime}$. Consequently we cannot see any structure below that size. The fact that our peak falls with $7^{\prime \prime}$ somewhat above the $5.5^{\prime \prime}$ resolution limit might indicate that the CLEAN beam used in the reduction of the interferometric data is not exactly Gaussian or slightly wider than computed. The whole map is taken with an interferometric mosaicing technique (see Testi \& Sargent 2000 for details) without a zero-spacing by complementary single dish observations. Thus the map cannot contain any structure on scales above the single pointing areas determined approximately by the size of the primary beam of the OVRO antennas of $73^{\prime \prime}$. This is in agreement with the lack of structure indicated by the $\Delta$-variance slope of -2 at these scales. The two limiting sizes are indicated by vertical lines in Fig. 8. Thus we may only discuss the range in between disregarding other information that is plotted in the interferometric map but that can eventually not be obtained from the observations.

The steepening of the $\Delta$-variance in the intermediate size range from $\alpha=-1.2$ to $\alpha=-2$ does qualitatively agree with the behaviour observed in most collapse simulations at small scales but does not match any of them quantitatively. For a detailed comparison the dynamic scale range covered in the simulations is still insufficient due to the periodic boundary conditions constraining the large scale behaviour. Hence, we can only conclude that the collapse models show the same general structure as the dust observations, indicating that they represent a realistic scenario but we cannot yet discriminate between different models using the observational data.

\subsection{Molecular line observations}

Bensch et al. (2001) provided a detailed $\Delta$-variance analysis of the density structure traced by observations in different $\mathrm{CO}$ isotopes for several molecular clouds with different states of star formation including quiescent clouds like the Polaris Flare and clouds with violent star formation like Orion A. They found for all molecular clouds a density structure approximately characterised by a power law $\Delta$ variance, with an exponent in the range $0.5 \leq \alpha \leq 1.3$. In the best studied cloud one smooth curve connects scales larger than $10 \mathrm{pc}$ (where turbulence presumably is driven) with the dissipation scale at 0.05 pc (where ambipolar diffusion processes become important). The positive slopes indicate that the density structure seen in the CO isotopes is dominated by large-scale modes. This result is consistent with purely supersonic turbulence and appears independent of the dynamical state of the molecular cloud regions studied, i.e. regardless whether the cloud forms stars or not.

This is somewhat surprising, since we expect that the density distribution in star-forming regions is dominated by the collapsing protostellar cores on small spatial scales. The $\Delta$-variance spectrum therefore should exhibit a $n e g$ ative slope as we demonstrate in Sect. 3. The molecular line results are also in obvious contradiction to the Serpens dust observations discussed above.

The explanation for the difference is hidden in the radiative transfer problem. A discussion of all major aspects of molecular line transfer in turbulent media is beyond the scope of this study and will be provided in a separate paper (Ossenkopf in prep.). Here, it is sufficient to concentrate on one effect - saturation at large optical depths. Molecular lines like the lower transitions of ${ }^{13} \mathrm{CO}$, frequently used to map the density profile of molecular clouds, become typically optically thick in the cores of clouds at densities in the order of $10^{5} \mathrm{~cm}^{-3}$. The exact value depends on the transition, the spatial configuration, temperatures, and the geometry of the radiation field but one can always assign a typical density range to the transition from the optically thin to the optically thick regime. This leads to a saturation of the line intensities in dense clumps so that the lines do not trace their internal structure but rather see clump surfaces. Moreover, the molecules tend to freeze out in dense dark regions (Kramer et al. 1999) amplifying the effect that the line brightness reflects only part of the column density in dense clumps.

As we do not want to treat the full radiative transfer problem here, we give only an estimate for the influence of optical depth effects by including a saturation limit into our computations. Because the simulations are scalefree and the typical saturation density varies for different molecules and transitions there is no particular density value to be used for this limit so that we have to play with different values.

Figure 9 illustrates the evolution of the large-scale driven model shown in Fig. 2a assuming now that all 

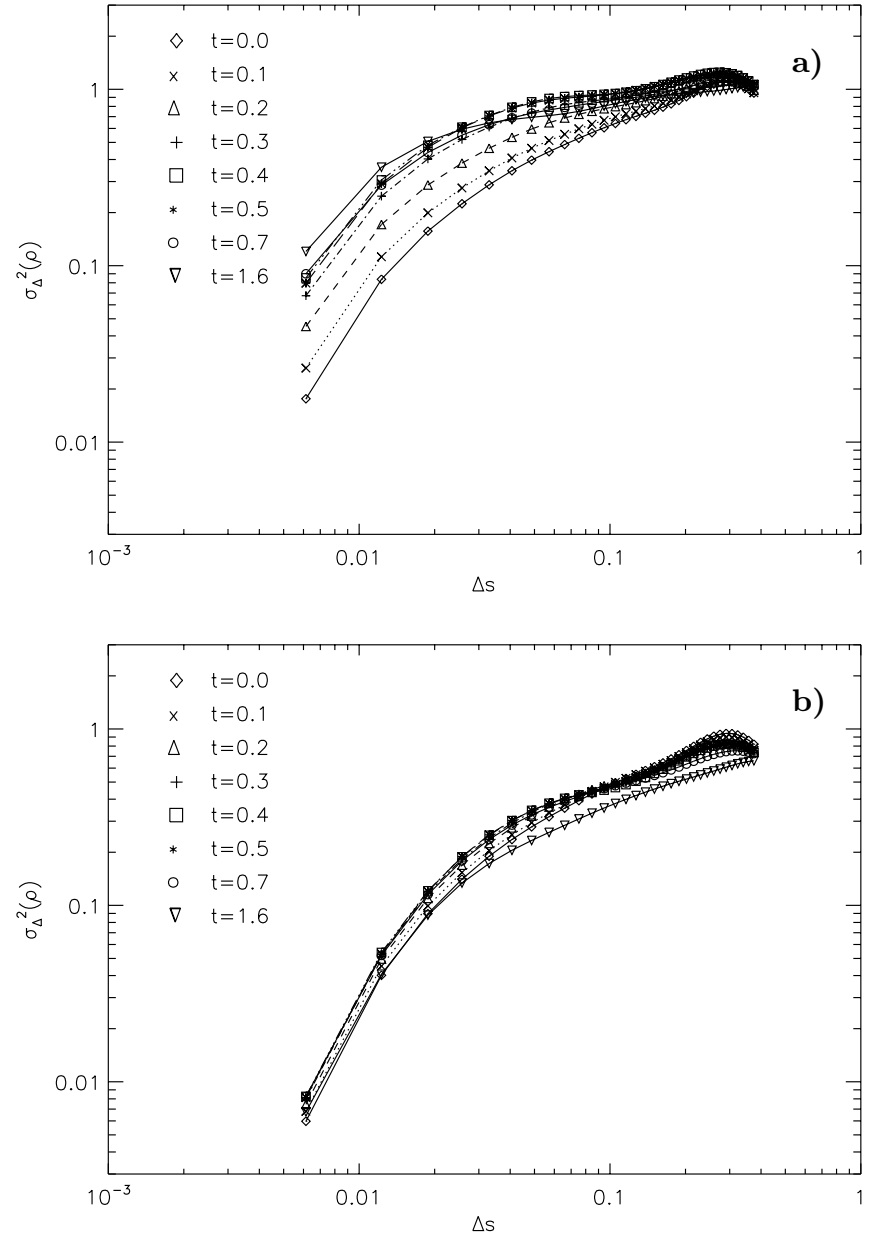

Fig. 9. Time evolution of the $\Delta$-variance for the large-scale driven model S01 when the density structure is assumed to saturate at densities of 240.0 a) and 24.0 b).

densities above a certain threshold are invisible so that they are equal to the value of the saturation density. In the upper plot we have chosen this limit to be the maximum density occurring in the original turbulent density distribution before gravitational collapse starts. This is 240 times the average density, i.e. a relatively large value compared to the dynamic range of molecular line observations. In the lower graph the saturation limit is reduced by a factor 10 .

Although only a relatively small fraction of the material appears at densities above the limit the influence on the $\Delta$-variance is dramatic. As the collapsing cores produce the relative enhancement of small scale structure their virtual removal by the saturation results in an almost constant $\Delta$-variance behaviour during the gravitational collapse. Even in the very conservative upper plot where we assume that all structures occurring in normal interstellar turbulence are still optically thin and only the collapsing cores become optically thick the $\Delta$-variance stays at a positive slope during the entire evolution. In the other case where optical depth effects are assumed to be more important the $\Delta$-variance remains at a fixed slope of 0.5 consistent with the molecular line observations of interstellar clouds.
Hence, optical depth effects can easily prevent the detection of gravitational collapse in molecular line observations, since they reproduce the $\Delta$-variance spectrum of a turbulent molecular cloud even if collapse has lead already to the formation of protostellar cores. Even in massively star-forming clouds most molecules used for mapping trace the diffuse density structure between Jeans-unstable collapsing protostellar cores. This gas is still dominated by interstellar turbulence. The density contrast in starforming molecular cloud regions simply exceeds the density range traceable by molecular transitions. Protostellar core densities are so high that ${ }^{13} \mathrm{CO}$ at best traces the outer envelope. Therefore both, star-forming and quiescent molecular clouds, exhibit very similar molecular line maps.

It is essential to resolve large density contrasts measuring the full density structure to study the influence of self-gravity and local collapse in star-forming clouds. This can be achieved using dust continuum emission. Indeed, the $3 \mathrm{~mm}$ continuum map of the Serpens cluster by Testi \& Sargent (1998) shows a density structure that is dominated by small scales as predicted by our collapse simulations. The drawback of dust emission observations is the inherent convolution of the density structure with the unknown temperature profile. Large dust extinction maps could circumvent this problem but require long integration times at NIR wavelengths to obtain a dense sampling with background stars (Lada et al. 1999).

\section{Summary}

In this paper we investigate the time evolution of the structural characteristics of self-gravitating supersonically turbulent gaseous systems using the $\Delta$-variance as statistical measure. We resolve the transition from purely hydrodynamic turbulence to gravitational collapse, the formation and mass growth of protostellar cores, and compare different models of driven and freely decaying selfgravitating turbulence with and without magnetic fields.

Contrary to what is observed for purely hydrodynamic turbulence, self-gravitating supersonic turbulence yields a density structure that contains most power on the smallest scales (i.e. in the collapsed objects) as soon as local collapse has set in. This happens in all self-gravitating turbulence models regardless of the presence or absence of magnetic fields. The $\Delta$-variance $\sigma_{\Delta}^{2}(n)$ exhibits a negative slope and peaks at small scales as soon as local collapse produces dense cores. This is in contrast to the case of non-self-gravitating hydrodynamic turbulence where $\sigma_{\Delta}^{2}(n)$ has a positive slope and the maximum at the largest scales. Our results can therefore be used to differentiate between different stages of protostellar collapse in starforming molecular clouds and to determine scaling properties of the underlying turbulent velocity field.

The effect of protostellar collapse, however, is not visible in molecular line maps of star-forming clouds, as all molecules trace only a limited dynamic range of densities. The density contrast in star-forming regions is much 
larger. ${ }^{12} \mathrm{CO}$ and ${ }^{13} \mathrm{CO}$ observations, for example, trace only the inter-core gas distribution and at best the outer parts of individual protostellar cores. Hence, the density structure seen in these molecules is indistinguishable for star-forming and non-star-forming regions.

As resolving high density contrasts is the key for detecting the effect of star formation in the $\Delta$-variance, we propose observations of dust continuum or of the dust extinction instead. These techniques do not have the same limitations of the dynamic range and are therefore better suited to quantitatively study the full density evolution during the star-formation process. This is confirmed by a first comparison of our models with the $3 \mathrm{~mm}$ dust continuum map taken by Testi \& Sargent (1998) in Serpens.

Acknowledgements. We thank Leonardo Testi and Anneila Sargent for kindly providing us the FITS file of their $3 \mathrm{~mm}$ continuum map of the Serpens core. Furthermore we are grateful to J. Ballesteros-Paredes, P. Bodenheimer, A. Burkert, C. Kramer, and M.-M. Mac Low for valuable discussions. VO was supported by the Deutsche Forschungsgemeinschaft through the grant SFB 494B. RSK acknowledges support by a Otto-Hahn-Stipendium of the Max-Planck-Gesellschaft and subsidies from a NASA astrophysics theory program supporting the joint Center for Star Formation Studies at NASA-Ames Research Center, UC Berkeley, and UC Santa Cruz. Computations presented here were performed at the GRAPE-Cluster at the Max-Planck-Institut für Astronomie in Heidelberg, at the Sterrewacht Leiden, at the Rechenzentrum Garching of the Max-Planck-Gesellschaft, and at the National Center for Supercomputing Applications (NCSA). ZEUS was used by courtesy of the Laboratory for Computational Astrophysics at the NCSA. This research has made use of NASA's Astrophysics Data System Abstract Service.

\section{References}

de Avillez, M. A. 2000, MNRAS, 315, 479

Ballesteros-Paredes, J., Hartmann, L., \& Vázquez-Semadeni, E. 1999a, ApJ, 527, 285

Ballesteros-Paredes, J., Vázquez-Semadeni, E., \& Scalo, J. 1999b, ApJ, 515, 286

Bate, M. R., Bonnell, I. A., \& Price, N. M. 1995, MNRAS, 277, 362

Bensch, F., Stutzki, J., \& Ossenkopf, V. 2001, A\&A, 336, 636

Benz, W. 1990, in The Numerical Modeling of Nonlinear Stellar Pulsations, ed. J. R. Buchler (Kluwer Acad. Publ., Dordrecht), 269

Clarke, D. 1994, National Center for Supercomputing Applications Technical Report
Ebisuzaki, T., Makino, J., Fukushige, T., et al. 1993, PASJ, 45, 269

Elmegreen, B. G. 1993, ApJ, 419, L29

Elmegreen, B. G. 2000, ApJ, 530, 277

Heitsch, F., Mac Low, M.-M., \& Klessen, R. S. 2001, ApJ, 547, 280

Klessen, R. S. 1997, MNRAS, 292, 11

Klessen, R. S. 2000, ApJ, 535, 869

Klessen, R. S. 2001, ApJ, 556, 837

Klessen, R. S., \& Burkert, A. 2000, ApJS, 128, 287

Klessen, R. S., \& Burkert, A. 2001, ApJ, 549, 386

Klessen, R. S., Heitsch, F., \& Mac Low, M.-M. 2000, ApJ, 535, 887

Kramer, C., Alves, J., Lada, C. J., et al. 1999, A\&A, 342, 257

Lada, C. J., Alves, J., \& Lada, E. A. 1999, ApJ, 515, 265

van Leer, B. 1977, J. Comp. Phys., 23, 276

Mac Low, M.-M. 1999, ApJ, 524, 169

Mac Low, M.-M., \& Ossenkopf, V. 2000, A\&A, 353, 339

Mac Low, M.-M., Klessen, R. S., Burkert, A., \& Smith, M. D. 1998, Phys. Rev. Lett., 80, 2754

Monaghan, J. J. 1992, ARA\&A, 30, 543

Mouschovias, T. C., \& Spitzer, L. 1976, ApJ, 210, 326

Ossenkopf, V., \& Mac Low, M.-M. 2001, A\&A, submitted

Ossenkopf, V., Bensch, F., Mac Low, M.-M., \& Stutzki, J. 1999, in The Physics and Chemistry of the Interstellar Medium, ed. V. Ossenkopf, J. Stutzki, \& G. Winnewisser (GCAVerlag Herdecke), 216

Ossenkopf, V., Bensch, F., \& Stutzki, J. 2000, in The Chaotic Universe, ed. V. G. Gurzadyan, \& R. Ruffini (World Sci.), 394

Padoan, P. 1995, MNRAS, 277, 337

Padoan, P., \& Nordlund, Å. 1999, ApJ, 526, 279

Scalo, J. M., Vázquez-Semadeni, E., Chappell, D., \& Passot, T. 1998, ApJ, 504, 835

Spaans, M., \& Silk, J. 2000, ApJ, 538, 115

Stone, J. M., \& Norman, M. L. 1992a, ApJS, 80, 753

Stone, J. M., \& Norman, M. L. 1992b, ApJS, 80, 791

Stone, J. M., Ostriker, E. C., \& Gammie, C. F. 1998, ApJ, 508, L99

Stutzki, J., Bensch, F., Heithausen, A., Ossenkopf, V., \& Zielinsky, M. 1998, A\&A, 336, 697

Sugimoto, D., Chikada, Y., Makino, J., et al. 1990, Nature, 345, 33

Testi, L., \& Sargent, A. 1998, ApJ, 508, L91

Testi, L., \& Sargent, A. 2000, in Imaging at Radio through Sub-millimeter wavelengths, ed. J. Mangum, S. Radford, ASP Conf. Ser., 217, 283

Vázquez-Semadeni, E. 2000, in The Chaotic Universe, ed. V. G. Gurzadyan, R. Ruffini (World Sci.), 379

Williams, J. P., Blitz, L., \& McKee, C. 2000, in Protostars and Planets IV, ed. V. Mannings, A. P. Boss, \& S. S. Russell, 97

Zielinsky, M., \& Stutzki, J. 1999, A\&A, 347, 633 